\title{
Chaotic Scattering with Resonance Enhancement
}

\author{
B. Elattar:日, V. Kagalovsky, and H.A. Weidenmüller \\ Max-Planck-Institut für Kernphysik, 69029 Heidelberg, Germany
}

(March 7, 2018)

\begin{abstract}
The passage of light or of electrons through a disordered medium is modified in the presence of resonances. We describe a simple model for this problem, and present first results.
\end{abstract}

\section{INTRODUCTION AND MOTIVATION}

During the last decade, many phenomena related to the passage of waves through a disordered medium have been intensely studied. This holds both for electrons, i.e. for the amplitude waves of the Schrödinger equation, and for light, i.e. for classical electromagnetic waves. As examples, we mention conductance fluctuations in mesoscopic probes, and weak and strong localization effects including the enhanced backscattering of light reflected from a disordered medium. For electrons, disorder is typically caused by scattering from impurities. For light, disorder is often produced artificially: A powder is immersed into some liquid. Random scattering occurs if the indices of refraction of powder and liquid are sufficiently different.

The present paper has been motivated by an experiment performed several years ago [1] on the scattering of light by a disordered medium. In this experiment, the powder used $\left(\mathrm{TiO}_{2}\right)$ consisted of grains with a size distribution centered around a diameter of $220 \mathrm{~nm}$. For such granules, a Mie resonance occurs close to the wavelength $\lambda \sim 630 \mathrm{~nm}$ of the laser light used in the experiment. This led to a resonance enhancement of the scattering. The 
effects of such enhancement were seen by comparing the diffusion constant $D$ (determined from the intensity autocorrelation function versus frequency of the transmitted light), and the transport mean free path $l$ (determined either from weak localization, i.e. from enhanced backscattering, or from the dependence of the transmission intensity on the length $L$ of the disordered slab). One expects $D$ and $l$ to obey the relation $D=1 / 3 v_{E} l$, with $v_{E}$ the energy transport velocity through the disordered medium. In the present case, the ratio $3 D / l$ yielded a value $v_{E}=(5 \pm 1) 10^{7} \mathrm{~m} \mathrm{~s}^{-1}$ for the transport velocity $v_{E}$ which is about an order of magnitude smaller than the phase velocity. This surprising result has been understood both for small concentration [1] and, more recently, also for strong concentration [2] of the scatterers. Qualitatively speaking, the transport velocity is reduced because on its way through the medium, the energy is stored for some time in the Mie resonances.

In this paper, we study the passage of waves through a medium with randomly positioned resonant scatterers from the point of view of random matrix theory (RMT). To the best of our knowledge, this has not been done before. Our motivation is the following. First, the approach taken in refs. [1] and [2] is not entirely systematic. Indeed, it combines results of diagrammatic impurity perturbation theory (used for analysing the intensity autocorrelation function) with the use of the Bethe-Salpeter equation [1], or of a mean-field approximation [2], for the calculation of the transport velocity $v_{E}$. It is our hope that a suitable modification of RMT (capable of accounting for the presence of resonance scattering) may yield a uniform account for both, average and fluctuation properties. This is all the more desirable since during the last decade, RMT has developed into one of the most important tools for investigating wave propagation in disordered media. The problem studied in this paper calls for an extension of existing RMT and would thus widen the scope of this theory. Second, RMT would make it conversely possible to put the present problem into a wider context, and to study, for instance, the effect of resonances on localization properties.

We have embarked on this project only recently. The present paper is, therefore, no more than a work-in-progress report. We simplify the problem by focussing on scalar waves with a simple dispersion relation (i.e., electrons), thus postponing the description of the 
propagation of light waves to a later stage. The RMT model with resonances is introduced in section II]. In section [II], Efetov's supersymmetric generating functionals [3] in the version of ref. [4] are used to handle the technical problems resulting from the model. First results obtained in the simplest case are presented in section $\mathbb{}$.

\section{THE MODEL}

To motivate the model, and to identify the relevant parameters, we begin with a few qualitative considerations. We consider a volume $V$ of liquid containing a volume concentration $\mu$ (defined as volume of grains per total volume) of resonant powder. To simplify the problem, we assume that all grains (i.e., all Mie scatteres) are exactly equal, each having volume $\Phi$, and resonance frequency $\omega_{1}$. Then, the number $m$ of grains in $V$ is $m=\mu V / \Phi$. The total volume $m \Phi=\mu V$ occupied by the grains is denoted by $V_{\text {grain }}$, the remaining volume $V_{\text {rem }}$ left for the liquid is $V_{\text {rem }}=V(1-\mu)$.

We denote by $\rho_{L}^{(0)}(\omega)$ the density of states per unit volume in the liquid for a classical scalar wave of frequency $\omega$. We have [5]

$$
\rho_{L}^{(0)}(\omega)=\frac{\omega^{2}}{2 \pi^{2} v_{0}^{3} \hbar},
$$

where $v_{0}$ is the phase velocity of the wave in the liquid. For $\mu=0$, the total density of states in the liquid is $\rho_{L}=\rho_{L}^{(0)}(\omega) V$. For $\mu \neq 0$ we only have the remaining volume $V_{\text {rem }}$ at our disposal, so that $\rho_{L, r e m}=\rho_{L}^{0} V_{\text {rem }}=\rho_{L}^{0} V(1-\mu)$. For later use, we note that for a vessel of length $L=17 \mu \mathrm{m}$ and area $A=610^{3}(\mu \mathrm{m})^{2}$, for $v_{0}=c$ (the velocity of light in vacuum), and for a wave length $\lambda=630 \mathrm{~nm}$, the mean level spacing $d_{L}=\rho_{L}^{-1}$ is about $410^{-7} \mathrm{eV}$.

With increasing concentration of scatterers, the remaining volume shrinks, and with it the level density of the liquid. We may ask: Where is the "missing level density" $\rho_{L}^{0} V \mu$ ? To answer this question, we consider a single grain. In this grain, there are many Mie resonances, with a level density $\rho_{\text {Mie }}$ which is proportional to $\Phi$ (so that $\rho_{\text {Mie }}=\rho_{\text {Mie }}^{0} \Phi \ll \rho_{L}$ ). The total level density in all $m$ grains is $m \rho_{\text {Mie }}=\rho_{M i e}^{0} m \Phi=\rho_{M i e}^{0} V \mu$. This is exactly the missing 
contribution provided we have $\rho_{L}^{0}=\rho_{\text {Mie }}^{0}$. In practice, this condition will not hold, because the level density depends on the index of refraction of the medium. For purposes of the present qualitative discussion, we disregard this fact.

We see that the immersion of the grains in the liquid causes a concentration of level density at the position of the Mie resonances: While $\rho_{L}$ is lowered from $\rho_{L}^{0} V$ to $\rho_{L}^{0} V(1-\mu)$, $\rho_{\text {Mie }}$ increases from zero to $\rho_{M i e}^{0} V \mu$. We note that on the scale of the mean level spacing $d_{L}=1 /\left(\rho_{L}^{0} V(1-\mu)\right)$ in the liquid, $\rho_{M i e}$ is not smooth but sharply peaked at the position of the Mie resonances. The area under each peak is $m$. We also note that typically, $m \gg 1$ : For a concentration of 1 percent, a grain size of $200 \mathrm{~nm}$, and a volume of $10^{-13} \mathrm{~m}^{3}$, we have $m \sim 10^{5} \quad$ !

We expect the shape of the peak of $\rho_{\text {Mie }}$ to be Lorentzian, with a width $\Gamma$ given by the decay width of the individual Mie resonances (we assume that the concentration is sufficiently low so that the resonances do not "talk" to each other). Noticeable optical effects can be expected only if the peak height $2 m /(\pi \Gamma)$ is at least comparable with $\rho_{L}$, i.e. for $m d_{L} \sim \Gamma$, or for $\mu \geq \rho_{L}^{(0)} \Gamma$. When is this condition met? For light, we typically have $\Gamma \sim 0.1 \mathrm{eV}$. Using this figure and the values for $\rho_{L}$ and $\Phi$ given above, we find $\mu \geq 2$ percent.

Naturally, these considerations are only semiquantitative. Nonetheless, they establish the following picture. For grain concentrations in the percent region, there occurs a significant local enhancement of the level density at the characteristic frequency $\omega_{1}$ of the Mie scatterers, with a width given by the decay width $\Gamma$ of the Mie resonance. Typically, this width is very much larger than the mean level spacing $d_{L}$ in the liquid. For frequencies $\omega$ differing from the Mie resonance frequency $\omega_{1}$ by more than a few units of $\Gamma / \hbar$, the wave incident on a grain does not excite the Mie resonance, and the scattering by the wave on the grains is a totally random process kin to diffusive impurity scattering. As $\omega$ approaches $\omega_{1}$, resonant scattering will be superposed onto this random process. Even for $\omega=\omega_{1}$, there will still be random scattering since upon hitting a Mie scatterer, only part of the incident wave will excite the resonance, the remainder undergoing hard-sphere reflection.

How can we model this situation in the framework of RMT? We recall how diffusive 
scattering in the absence of Mie resonances is modelled in the framework of RMT, for a quasi one-dimensional system of length $L$ [6]: The system is considered as consisting of many longitudinal slices of length $l \ll L$. Within each slice, the Hamiltonian is modelled as a random Hamiltonian matrix (a member of the random-matrix ensemble with the proper symmetry). Neighboring slices are coupled by Gaussian-distributed uncorrelated random matrix elements. The first and the last slice are coupled to the leads or, equivalently, to the channels. Elements of the scattering matrix connecting incident and outgoing channels define the conductance, i.e. the transmission through the disordered region.

With proper modifications, we use this model also for the present problem. To begin with, we consider only Schrödinger waves. The modifications caused by the different form of the Hamiltonian for light waves will be addressed at a later stage. We retain the idea of a division of the quasi one-dimensional system into slices. To account for the presence of Mie scatterers, the form of the Hamiltonian has to be modified: Within each slice, our Hamiltonian $\mathbf{H}$ is a matrix of dimension $N+m_{S}$ where $m_{S}$ is the number of Mie scatterers within each slice. The Hamiltonian $\mathbf{H}$ contains a purely random part (a matrix $H_{G O E}$ of dimension $N$ belonging to the Gaussian orthogonal ensemble [GOE]), a diagonal part of dimension $m_{S}$ with diagonal elements $E_{1}=\hbar \omega_{1}$ corresponding to the presence of $m_{S}$ Mie scatterers with equal resonance frequencies $\omega_{1}$, and a rectangular matrix $V$ which couples the $m_{S}$ resonaces to $H_{G O E}$. Since we will focus on the Hamiltonian for a single slice in all that follows, we replace $m_{S}$ by $m$ for simplicity. With $I_{m}$ the $m$-dimensional unit matrix and with $T$ denoting the transpose, we thus have

$$
\mathbf{H}=\left(\begin{array}{cc}
H_{G O E} & V \\
V^{T} & E_{1} \times I_{m}
\end{array}\right) .
$$

The dimension $N$ of $H_{G O E}$ is taken to infinity at the end of the calculation. The variance of the matrix elements $H_{G O E}$ is normalized as usual: The ensemble average of $\left(H_{G O E}\right)_{\mu \nu}\left(H_{G O E}\right)_{\mu^{\prime} \nu^{\prime}}$ is given by $\left(\lambda^{2} / N\right)\left(\delta_{\mu, \mu^{\prime}} \delta_{\nu, \nu^{\prime}}+\delta_{\mu, \nu^{\prime}} \delta_{\nu, \mu^{\prime}}\right)$. Here, $\lambda$ (not to be confused with the wave length of the scattered light) has the dimension of an energy and determines the average level spacing $d_{S}$ in each slice. For $m=0$, the average level density obeys the 
semicircle law, and the dependence of $d_{S}$ on energy $E$ is given by $\pi \lambda / N \sqrt{1-(E /(2 \lambda))^{2}}$. For $m \neq 0$, we expect the average level density to deviate from the semicircle law by aquiring an additional peak at energy $E_{1}$. The width of the peak will be determined by the matrix $V$. By assuming the $m$-dimensional matrix in the lower right-hand block to be diagonal, we implement the assumption that the $m$ resonances do not "talk" to each other, i.e., are not coupled directly. By the same token, we assume that resonances in different slices are not directly coupled to each other. Both assumptions are obviously valid only for a sufficiently low concentration of Mie scatterers. Because of this assumption, the rectangular matrix $V$ can, without loss of generality, be taken to be diagonal. Indeed, $V$ can be written in the form $O_{1} V_{D} O_{2}^{T}$ where $V_{D}$ is diagonal, and where $O_{1}$ and $O_{2}$ are orthogonal matrices of dimension $N$ and $m$, respectively. Transforming $\mathbf{H}$ with the orthogonal matrix $O=\operatorname{diag}\left(O_{1}, O_{2}\right)$, and absorbing the matrix $O_{1}$ into $H_{G O E}$ by virtue of the orthogonal invariance of the GOE, we obtain a new Hamiltonian of the form of Eq. (2), but with $V$ replaced by the diagonal matrix $V_{D}$. Since we assume all scatterers to be identical, we take $V_{D}$ to be a multiple of the unit matrix, $V_{D}=v \times I_{m}$. This completes the definition of the model.

\section{SUPERSYMMETRIC FORMALISM}

So far, we have only considered the simplest apects of the model defined in section $\amalg$ : For technical reasons, we have replaced the GOE by the unitary ensemble (GUE), and we have calculated the mean level density and the two-point level correlation function. This was done to make sure that the model behaves as we expect, and to gain experience with a Hamiltonian of the form of Eq. (2). We have used the supersymmetry technique of refs. [3:4]. We omit all details, use the notation of ref. [4], and give only a few results which display characteristic differences between the usual procedure, and the present model.

After averaging and the Hubbard-Stratonovich transformation, the exponent of the generating functional for the two-level correlation function (with energies $E \pm \omega$ ) has the form 


$$
\left.-\frac{N}{2 \lambda} \operatorname{trg} \sigma^{2}-\operatorname{trg} \log \left(\begin{array}{ccc}
E-\lambda \sigma-\omega \Lambda-J & 0 & -V \\
0 & E-\lambda \sigma-\omega \Lambda-J & 0 \\
-V & 0 & E-E_{1}-\omega \Lambda-J
\end{array}\right)\right\} m
$$

The symbols $J$ stand for the source terms. The dimensions of the block matrices are indicated at the side, and $V$ stands for $v \times I_{m}$. Variation with respect to $\sigma$ leads to the saddle-point equation

$$
\frac{\sigma}{\lambda}=\left(1-\frac{m}{N}\right) \frac{1}{E-\lambda \sigma}+\frac{m}{N} \frac{E-E_{1}}{(E-\lambda \sigma)\left(E-E_{1}\right)-v^{2}}
$$

As usual, we have assumed that $\omega \sim 1 / N$, and we have omitted the (Infinitesimal) source terms. We take the limit $N \rightarrow \infty$ and keep $m$ fixed. This yields the standard saddle-point equation

$$
\frac{\sigma}{\lambda}=\frac{1}{E-\lambda \sigma}
$$

with solution

$$
\sigma=\frac{E}{2 \lambda}+i \Delta
$$

where $\Delta=\sqrt{1-(E / 2 \lambda)^{2}}$. In contrast to the usual case, the average level density is no longer given by the saddle-point solution of Eq. (6) but attains an extra piece which is due to the last block in the matrix displayed in Eq. (3). Likewise, the two-point function differs from the regular form, and has to be calculated by keeping carefully track of the extra terms appearing in Eq. (3). The results are as follows.

For the average density of states, we define the level density for the Hamiltonian $\mathbf{H}$ as $\rho(E)=\sum_{i} \delta\left(E-E_{i}\right)$. Let a bar denote the ensemble average. Then, we find

$$
\overline{\rho(E)}=\frac{\Delta(E) N}{\lambda \pi}+m \frac{\Gamma \Delta(E) / \pi}{\left(E-E_{1}-(E / 2 \lambda) \Gamma\right)^{2}+(\Gamma \Delta)^{2}},
$$

where $\Gamma=v^{2} / \lambda$ defines the effective width $\Gamma \Delta$ of the Lorentzian. The form of Eq. (17) is expected. Indeed, $\pi \lambda /(N \Delta)$ is the mean level spacing $d$ of the GUE and is to be identified 
with $d_{L}$, the mean level spacing in the liquid. The last term in Eq. (7) has area $m$, peak height $m /(\pi \Gamma \Delta)$, and Lorentzian shape, all as anticipated in section III. The occurrence of the factor $\Delta$ reflects the semicircle law for the GUE level density, and the factor $E /(2 \lambda)$ multiplying $\Gamma$ is an associated level shift which vanishes in the center of the semicircle where $E=0$. The form of $\Gamma$ is also in keeping with expectations. Indeed, we recall that the GUE matrix elements have variance $\lambda^{2} / N$. We expect the matrix elements $v$ occurring in Eq. (雨) to scale similarly with $N$, so that $v^{2}=\alpha \lambda^{2} / N$ where $\alpha$ is independent of $N$. Then, $\Gamma=\alpha \lambda / N$ so that $\Gamma$ is given as $\alpha \pi / \Delta$ times the mean level spacing of the GUE. For light scattering, $\alpha \gg 1$ although we also consider the case $\alpha \sim 1$ in this paper.

The Lorentzian in Eq. (7) is plainly a superposition of $m$ individual resonances. It may come as a surprise that the indirect interaction between resonances caused by their joint coupling to the random Hamiltonian does not cause any level repulsion between the $m$ Lorentzians. To clarify this issue, we have solved the saddle-point equation (四) to next order in $N^{-1}$. We find that this leads to an energy-dependent broadening of the Lorentzian in Eq. (7) which obviously is of order $N^{-1}$ relative to the terms we have kept. We conclude that within the approximations leading to Eq. (7), the indirect coupling between resonances is negligible.

Since the presence of the $m$ resonances at energy $E_{1}$ destroys translational symmetry, the evaluation of the level-level correlation function is slightly more complicated than is the case usually. We calculate

$$
C(E, \omega)=\overline{\rho(E+\omega) \rho(E-\omega)}-\overline{\rho(E+\omega)} \overline{\rho(E-\omega)} .
$$

The disconnected terms cancel as usually if the "boundary terms" [7] or "Efetov-Wegner" terms [4] are taken into account. The remaining integrand contains an exponential with argument

$$
-2 i \pi \omega / d \operatorname{trg}\left(1+\alpha_{1}\right)+m \operatorname{trg} \log \left(\left(E-E_{1}-E \Gamma /(2 \lambda)\right)^{2}+\Delta^{2} \Gamma^{2}-\omega^{2}-2 i \omega \Gamma \Delta\left(1+\alpha_{1}\right)\right) .
$$


Here, $\alpha_{1}$ is defined as in ref. [四]. It is easily seen that for $\omega \ll \Gamma$, this expression reduces to $-2 i \pi \omega / d_{\text {eff }}(E) \operatorname{trg}\left(1+\alpha_{1}\right)$ where $d_{\text {eff }}(E)$ is the inverse of the average level density $\overline{\rho(E)}$ given in Eq. (7). By virtue of the integration over the variables in $\alpha_{1}$, the $\omega$-dependence of $C(E, \omega)$ is effectively cut off at $\omega \sim d_{e f f}$. For values of $\Gamma \gg d_{e f f}$, the normalized two-point autocorrelation function therefore essentially coincides with the one for the pure GUE except for a rescaling of the average level spacing. Differences can occur only in the wings of the distribution. or in cases where $\Gamma \sim d_{e f f}$. The figures presented in the next section are based on the exact form of $C(E, \omega)$ given by

$$
\begin{aligned}
& C(E, \omega)=\overline{\rho(E+\omega) \rho(E-\omega)}=\operatorname{Re}\left[\frac{1}{2 d^{2}}\left(\frac{i D}{2 \pi \omega}\right)^{-m+1} \exp (2 \pi \omega D / d) \Gamma(-m+1,2 \pi \omega(D-i) / d)\right. \\
& \times \int_{-1}^{1} d \lambda_{2}\left(i D+\lambda_{2}\right)^{m} \exp \left(-2 i \pi \omega \lambda_{2} / d\right)--\frac{m}{2 \pi d}\left(\frac{i D}{2 \pi \omega}\right)^{-m} \exp (2 \pi \omega D / d)\left(\frac{D}{\omega}+\frac{1}{\Gamma \Delta}\right) \\
& \times \Gamma(-m, 2 \pi \omega(D-i) / d) \int_{-1}^{1} d \lambda_{2}\left(i D+\lambda_{2}\right)^{m-1} \exp \left(-2 i \pi \omega \lambda_{2} / d\right)--\frac{m^{2}}{8 \pi^{2}}\left(\frac{i D}{2 \pi \omega}\right)^{-m-1} \frac{D^{2}+1}{\omega^{2}} \\
& \left.\times \exp (2 \pi \omega D / d) \Gamma(-m-1,2 \pi \omega(D-i) / d) \int_{-1}^{1} d \lambda_{2}\left(i D+\lambda_{2}\right)^{m-2} \exp \left(-2 i \pi \omega \lambda_{2} / d\right)\right],
\end{aligned}
$$

where $\Gamma(a, b)$ is the Gamma function, and where

$$
D=\left[\left(E-E_{1}-(E / 2 \lambda) \Gamma\right)^{2}+(\Gamma \Delta)^{2}-\omega^{2}\right] /(2 \omega \Gamma \Delta) .
$$

The remaining integrals in Eq. (10) can be calculated analytically for every $m$. The resulting expressions are too lengthy to be given here.

In the sequel, we also consider the normalized quantity

$$
C_{\text {nor }}(E, \omega)=\frac{C(E, \omega)}{\rho(E-\omega) \rho(E+\omega)} .
$$

\section{RESULTS}

We have commented above on the form of the average level density given in Eq. (7); these comments will not be repeated here. Rather, we focus attention on the normalized level-level correlation function defined in Eq. (12). With all energies scaled in units of the GUE mean level spacing $d=\pi \lambda / N$ at the center of the unit circle, we present in Figure 1 
the function $C_{n o r}$ versus $\omega / d$ for parameter values as given in the caption. For comparison, we also show the standard GUE level-level correlation function $\sin ^{2}\left(2 \pi \omega / d_{\text {eff }}\right) /\left(2 \pi \omega / d_{\text {eff }}\right)^{2}$. We note that in the figure, we have chosen $\Gamma=0.3 d$ so that differences between the two functions should be visible in the wings. As anticipated above, we find that the two functions nearly coincide within the peak. While the GUE correlation function is positive semidefinite everywhere, the function $C_{\text {nor }}$ oscillates in the wings around zero.

In Figure 2, we show $C_{n o r}$ for the case $\Gamma=3 d$. Here, there is no discernible difference between this function and the standard GUE autocorrelation function $\sin ^{2}\left(2 \pi \omega / d_{e f f}\right) /\left(2 \pi \omega / d_{e f f}\right)^{2}$ provided that the latter is scaled with $d_{e f f}$. The second function in Figure 2 with the wide peak shows the GUE correlation function evaluated without rescaling, i.e. the function $\sin ^{2}\left(2 \pi \omega / d /(2 \pi \omega / d)^{2}\right.$. The difference between both functions is substantial.

In summary, we have presented first analytical results for a random-matrix model describing wave propagation in a random medium with resonant scattering. We are confident that this work will produce further interesting results.

\section{ACKNOWLEDGMENTS}

V. K. gratefully acknowledges the support of a MINERVA Fellowship. B. E. wishes to thank Prof. A. Nourreddine for valuable discussions. 


\section{REFERENCES}

* Also at Université Chouaïb Doukkali, Faculté des Sciences, El Jadida, Morocco.

[1] M. van Albada, B. A. van Tiggelen, A. Lagendijk, and A. Tip, Phys. Rev. Lett. 66 (1991) 3132.

[2] K. Busch and C. M. Soukoulis, Phys. Rev. Lett. 75 (1995) 3442.

[3] K. B. Efetov, Adv. Phys. 32 (1983) 53.

[4] J. J. M. Verbaarschot, M. R. Zirnbauer, and H. A. Weidenmüller, Phys. Rep. 129 (1985) 387.

[5] P. Sheng, Introduction to Wave Scattering, Localization, and Mesoscopic Phenomena, Academic Press, New York (1995).

[6] S. Iida, H. A. Weidenmüller, and J. Zuk, Phys. Rev. Lett. 64 (1990) 583 and Ann. Phys. (N.Y.) $200(1990) 219$.

[7] M. R. Zirmbauer and F. D. M. Haldane, preprint cond-mat/9504108 (1995). 


\section{FIGURES}

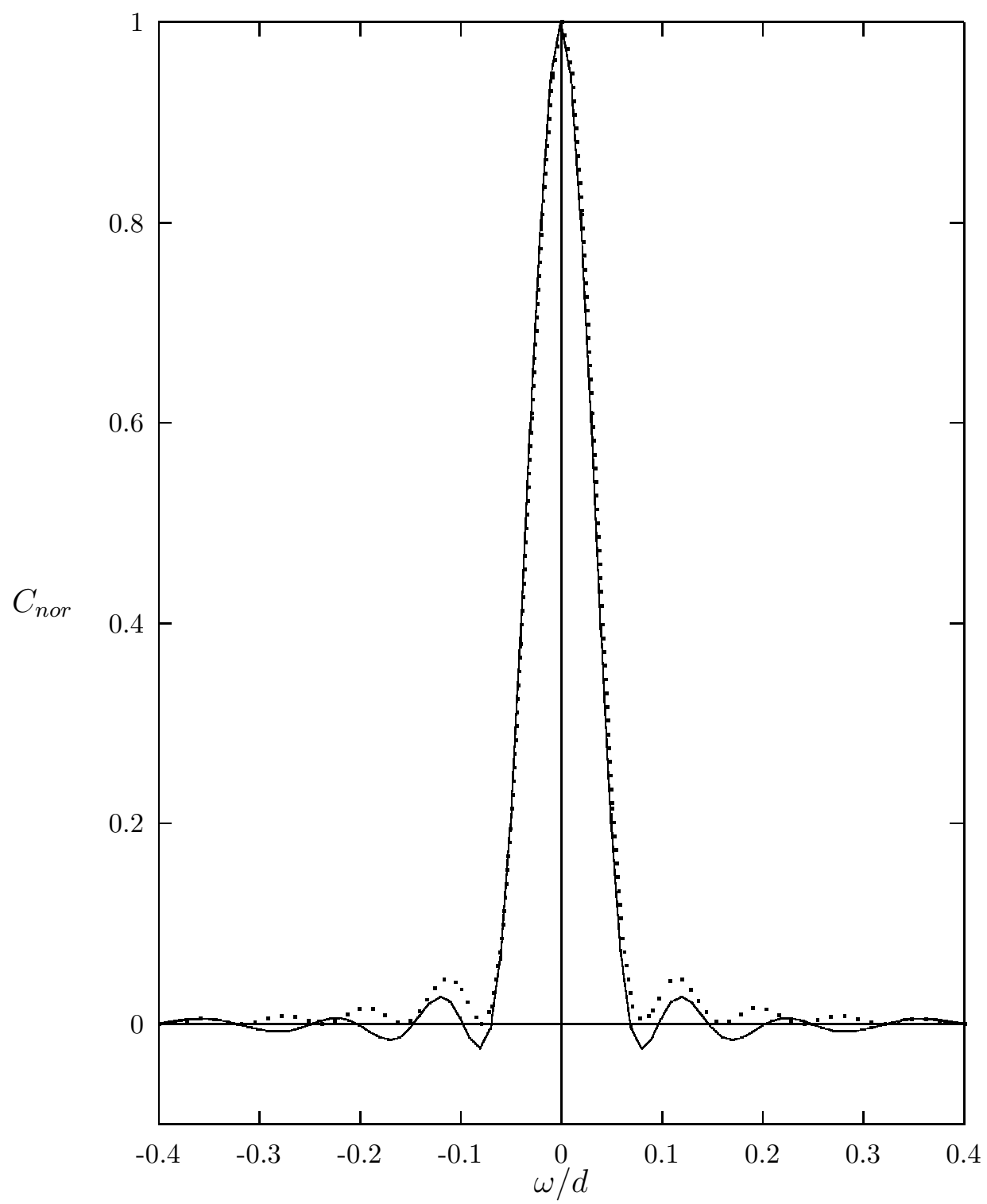

Fig.1. The normalized level-level correlation function of Eq. (8) for $m=5, \Gamma=0.3 d$, $E=E_{1}=0$ (full line) and $\sin ^{2}\left(2 \pi \omega / d_{\text {eff }}\right) /\left(2 \pi \omega / d_{\text {eff }}\right)^{2}$ with $d_{\text {eff }}=d /(1+m /(\pi \Gamma))($ dotted line). 


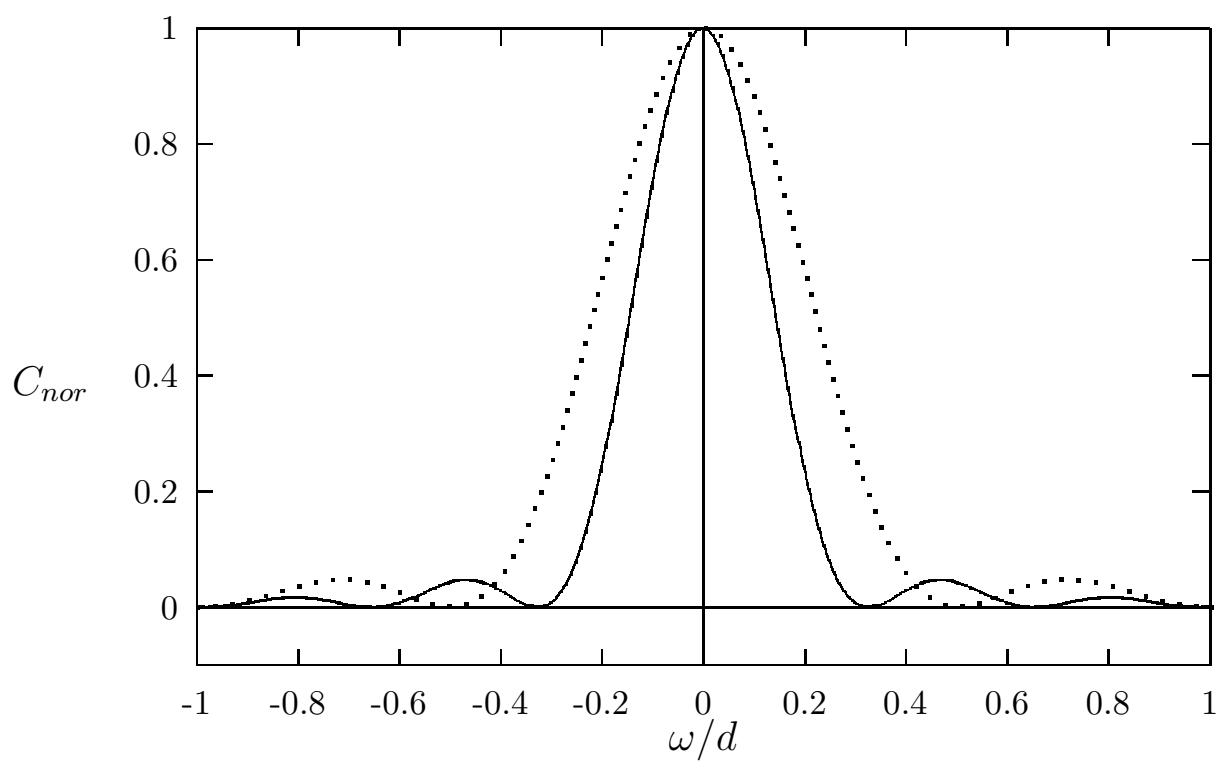

Fig.2. The normalized level-level correlation function of Eq. (8) for $m=5, \Gamma=3 d$, $E=E_{1}=0$ (which coincides almost exactly with $\sin ^{2}\left(2 \pi \omega / d_{\text {eff }}\right) /\left(2 \pi \omega / d_{\text {eff }}\right)^{2}$ with $\left.d_{\text {eff }}=d /(1+m /(\pi \Gamma))\right)$ (full line) and the standard normalized GUE correlation function $\sin ^{2}(2 \pi \omega / d) /(2 \pi \omega / d)^{2}$ (dotted line). 